\title{
Elastodynamics model updating for the monitoring of reinforced concrete beam: methodology and numerical implementation
}

\author{
J. Waeytens ${ }^{1, a}$, V. le Corvec ${ }^{2, b}, P$. Lévèque ${ }^{1, c}, D$. Siegert ${ }^{1, d}$ and F. Bourquin ${ }^{1, e}$ \\ ${ }^{1}$ University Paris-Est, IFSTTAR, Bvd Newton, F-77747 Champs sur Marne, France \\ ${ }^{2}$ Numerical Engineering \& Consulting Services, 196, rue Houdan, F-92330 Sceaux, France \\ ajulien.waeytens@ifsttar.fr, ${ }^{b}$ veronique.lecorvec@necs.fr, ${ }^{c}$ philippe.leveque@ifsttar.fr, \\ dominique.siegert@ifsttar.fr, efrederic.bourquin@ifsttar.fr
}

Keywords: inverse problem, optimal control, adjoint problem, structural dynamics.

\begin{abstract}
Reinforced concrete beams are widely employed in civil engineering structures. To reduce the maintenance financial cost, structure damages have to be detected early. To this end, one needs robust monitoring techniques. The paper deals with the identification of mechanical parameters, useful for Structural Health Monitoring, in a 2D beam using inverse modeling technique. The optimal control theory is employed. As an example, we aim to identify a reduction of the steel bar cross-section and a decrease of the concrete Young modulus in damaged areas. In our strategy, the beam is instrumented with strain sensors, and a known dynamic load is applied. In the inverse technique, two space discretizations are considered: a fine dicretization (h) to solve the structural dynamic problem and a coarse discretization $(\mathrm{H})$ for the beam parameter identification. To get the beam parameters, we minimize a classical data misfit functional using a gradient-like algorithm. A low-cost computation of the functional gradient is performed using the adjoint equation. The inverse problem is solved in a general way using engineer numerical tools: Python scripts and the free finite element software Code Aster. First results show that a local reduction of the steel bar cross-section and a local decrease of concrete Young modulus can be detected using this inverse technique.
\end{abstract}

\section{Introduction}

Reinforced concrete beams are widely employed in civil engineering structures. The maintenance of such structures is a central issue. To reduce maintenance costs, the damaged beams in the structure have to be identified early. To this end, the beams should be monitored and non-destructive control methods should be used. For the damage detection techniques, we can distinguish model-free approaches [1] and inverse modeling techniques. Herein, we deal with the second category. In usual civil engineering calculations, one-dimensional models $[2,3]$ are widely used to simulate non-linear behaviors of reinforced concrete structures at a low computation cost. Nevertheless, in the present article, we focus on inverse problems based on two-dimensional (2D) beam modelisation and dynamic responses. As a matter of fact, by means of 2D modelisation, we can specify the 2D position of the sensors and we can precisely describe the geometry and the location of steel bars. Using dynamic strain responses, we are interested in identifying a local loss of steel bar cross-section and a local decrease of concrete Young modulus. For that, an inverse modeling technique based on the optimal control theory [4] is proposed. In the 2D elastodynamics direct problem, the dynamic loading is considered known. The inverse problem is solved in a general way using common engineering numerical tools: free finite element software Code_Aster [5], Python Numpy library and a Python mesh API provided by SALOME platform [6]. On a 2D concrete beam with a single steel bar, we show that a $25 \%$ local loss of the steel bar cross-section and a $25 \%$ local decrease of the concrete Young modulus can be detected with the proposed method. It corresponds to a $10 \%$ reduction of the equivalent flexural rigidity. The article is organized as follows: the inverse method is first presented; then, details about the numerical implementation are given and lastly, the inverse method is illustrated on a $2 \mathrm{D}$ concrete beam with a steel bar. 


\section{Methodology to identify a reduction of the steel bar cross sections and a local decrease of the concrete Young modulus in a 2D concrete beam}

We aim at updating the steel bar cross-section $S_{b}(\underline{x})$ and the concrete Young modulus $E_{c}(\underline{x})$ in a 2D concrete beam from the knowledge of measured strain such that it minimizes a data misfit functional. Note that the dynamic loading applied to the concrete beam is considered known. The minimization process is performed in an iterative way using the steepest descent direction (gradient method). At each iteration, the main steps are the followings:

- solve the direct problem (elastodynamics equations forward in time) considering the beam parameters $\mathrm{S}_{\mathrm{b}}(\underline{\mathrm{x}})$ and $\mathrm{E}_{\mathrm{c}}(\underline{\mathrm{x}})$ of the previous iteration;

- solve the adjoint problem (elastodynamics equations backward in time);

- compute the functional gradient using the direct and the adjoint states;

- update the steel bar cross-section $\mathrm{S}_{\mathrm{b}}(\underline{\mathrm{x}})$ and the concrete Young modulus $\mathrm{E}_{\mathrm{c}}(\underline{\mathrm{x}})$.

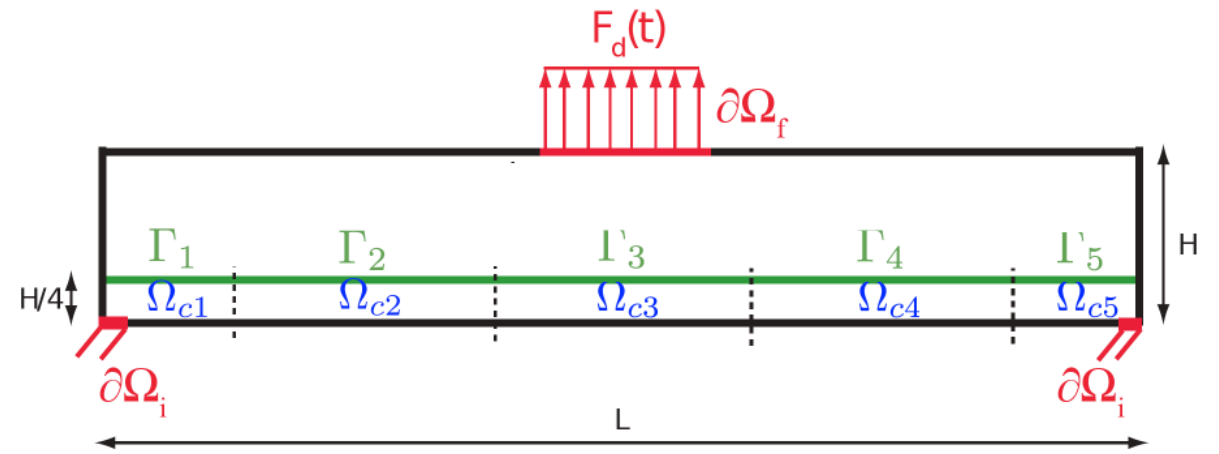

Fig. 1 - Concrete beam with a horizontal steel bar - Steel bar $\Gamma$ decomposed into 5 pieces $\left(n_{b}=5\right)$. Lower Concrete part $\Omega_{c}$ decomposed into 5 subdomains $\left(n_{c}=5\right)$.

\section{Definition of the beam parameters.}

To reduce the number of beam parameters to be determined, $S_{b}(\underline{x})$ and $E_{c}(\underline{x})$ are sought under the form:

$S_{b}(\underline{x})=\sum_{j=1}^{n_{b}} \check{S}_{b j} S_{b u d} \varphi_{j}^{b}(\underline{x}), E_{c}(\underline{x})=\sum_{j=1}^{n_{c}} \check{E}_{c j} E_{c u d} \varphi_{j}^{c}(\underline{x})$.

where:

- $n_{b}$ (resp. $n_{c}$ ) is the number of steel bar cross sections (resp. the number of concrete Young modulus) to be identified;

- $\mathrm{S}_{\text {bud }}(\underline{\mathrm{x}})$ (resp. $\mathrm{E}_{\mathrm{cud}}(\underline{\mathrm{x}})$ ) corresponds to the undamaged cross-section of the steel (resp. the undamaged Young modulus of the concrete);

- $\breve{S}_{\mathrm{bj}}$ (resp. $\breve{\mathrm{E}}_{\mathrm{cj}}$ ) represents the $\mathrm{j}^{\text {th }}$ normalized cross-section of the steel (resp. normalized Young modulus of the concrete) associated to the interval $\Gamma_{\mathrm{j}}\left(\operatorname{resp} . \Omega_{\mathrm{cj}}\right)$, i.e. the area where $\varphi_{\mathrm{j}}^{\mathrm{b}}(\underline{\mathrm{x}})$ (resp. $\varphi_{\mathrm{j}}{ }^{\mathrm{c}}(\underline{\mathrm{x}})$ ) is non-zero. $\breve{\mathrm{S}}_{\mathrm{bj}}$ and $\breve{\mathrm{E}}_{\mathrm{cj}}$ have to be determined using the inverse modeling technique;

- $\varphi_{\mathrm{j}}{ }^{\mathrm{b}}(\underline{\mathrm{x}})$ and $\varphi_{\mathrm{j}}^{\mathrm{c}}(\underline{\mathrm{x}})$ are basis functions on a coarse grid mesh $(\mathrm{H})$. They are a priori known. In practice, $\varphi_{j}{ }^{b}(\underline{x})\left(\operatorname{resp} . \varphi_{j}^{c}(\underline{x})\right)$ is a constant basis function equal to 1 over the interval $\Gamma_{j}$ (resp. in the subdomain $\Omega_{\mathrm{cj}}$ ) and equal to 0 elsewhere.

\section{Direct problem.}

In the present study, the shear work and the bending work in the steel bar are neglected. We also neglect the quantity of acceleration in rotation in the steel bar. We suppose a perfect adhesion between the steel bar and the concrete. We seek the displacement field $\underline{u}$ such that:

$\underline{u} \in \mathcal{U}_{0}=\left\{\underline{u}^{*} \in H_{1}(\Omega) \underline{u}^{*}=\underline{0}\right.$ on $\left.\partial \Omega_{i}\right\}$, 


$$
\begin{aligned}
\int_{\Omega} \rho_{c} \underline{\ddot{u}}^{*} d \Omega & +\int_{\Gamma} \rho_{b} S_{b} \underline{\ddot{u}}^{*} d \Gamma+\int_{\Omega} \varepsilon(\underline{u}): \mathrm{K}_{c} \varepsilon\left(\underline{u}^{*}\right) d \Omega+\int_{\Gamma} E_{b} S_{b} \frac{\partial u_{x}}{\partial x} \frac{\partial u_{x}^{*}}{\partial x} d \Gamma \\
& -\int_{\partial \Omega_{f}} \underline{F}_{d} \underline{u}^{*} \partial \Omega=0, \forall \underline{u}^{*} \in \mathcal{U}_{0} .
\end{aligned}
$$

The initial conditions are supposed to vanish.

\section{Data misfit functional.}

We seek the normalized steel bar cross-section $\breve{S}_{\mathrm{bj}}\left(\mathrm{j} \in\left\{1, \ldots, n_{b}\right\}\right)$ and the normalized concrete Young modulus $\breve{\mathrm{E}}_{\mathrm{cj}}\left(\mathrm{j} \in\left\{1, \ldots, n_{c}\right\}\right)$, minimizing the data misfit functional defined as:

$$
\begin{aligned}
J\left(\check{S}_{b}, \breve{E}_{c}\right)= & \frac{1}{2} \sum_{i=1}^{n_{s}} \int_{0}^{T}\left[\int_{\Omega} \varepsilon_{x x} \psi_{i}\left(\underline{x}-\underline{x}_{i}\right) d \Omega-\left(\varepsilon_{x x}^{m e s}\right)_{i}(t)\right]^{2} d t+\epsilon_{1} \frac{\alpha_{n 1}}{2} \int_{\Gamma}\left[\frac{S_{b}(\underline{x})}{S_{b u d}}-1\right]^{2} d \Gamma \\
& +\epsilon_{2} \frac{\alpha_{n 2}}{2} \int_{\Omega}\left[\frac{E_{c}(\underline{x})}{E_{c u d}}-1\right]^{2} d \Omega .
\end{aligned}
$$

where:

- $n_{s}$ is the number of strain sensors;

- $\quad\left(\varepsilon_{x x}^{m e s}\right)_{i}(t)$ is xx strain measured by sensor i located at $\underline{x}_{i}$;

- $\psi_{i}\left(\underline{x}-\underline{x}_{i}\right)$ is the spatial weight function associated to sensor $\mathrm{i}$.

In Eq. 3, one has a classical quadratic data misfit term and regularization terms. $\epsilon_{1}$ and $\epsilon_{2}$ are normalized regularization parameters. $\alpha_{n 1}$ and $\alpha_{n 2}$ ensure the physical homogeneity of the terms in the functional $J$. Herein, we take:

$\alpha_{n 1}=\frac{T}{L}, \alpha_{n 2}=\frac{T}{L^{2}}$.

\section{Adjoint problem, functional gradient and beam parameter update.}

To get the functional gradient at a low computational cost, we use the adjoint state. In this study, the adjoint problem corresponds to a backward elastodynamics problem (final conditions vanish). We seek the adjoint displacement field $\underline{\tilde{u}}$ such that:

$\underline{\tilde{u}} \in \mathcal{U}_{0}=\left\{\underline{u}^{*} \in H_{1}(\Omega) \underline{u}^{*}=\underline{0}\right.$ on $\left.\bar{\partial} \Omega_{i}\right\}$

$$
\begin{aligned}
\int_{\Omega} \rho_{c} \underline{\tilde{u}} \underline{u}^{*} d \Omega & +\int_{\Gamma} \rho_{b} S_{b} \underline{\ddot{\tilde{u}} u^{*}} d \Gamma+\int_{\Omega} \varepsilon(\underline{\tilde{u}}): \mathrm{K}_{c} \varepsilon\left(\underline{u}^{*}\right) d \Omega+\int_{\Gamma} E_{b} S_{b} \frac{\partial \tilde{u}_{x}}{\partial x} \frac{\partial u_{x}^{*}}{\partial x} d \Gamma \\
& -\sum_{i=1}^{n_{s}} \int_{\Omega}\left[\int_{\Omega} \varepsilon_{x x}(\underline{x}, t) \psi_{i}\left(\underline{x}-\underline{x}_{i}\right) d \Omega-\left(\varepsilon_{x x}^{m e s}\right)_{i}(t)\right] \psi_{i}\left(\underline{x}-\underline{x}_{i}\right) \frac{\partial u_{x}^{*}}{\partial x} d \Omega=0
\end{aligned}
$$

$\forall \underline{u}^{*} \in \mathcal{U}_{0}$.

Note that this problem can be solved using classical finite element codes.

From the direct and the adjoint states, we obtain the gradient of the functional, as follows:

$$
\begin{gathered}
\frac{\partial J}{\partial \check{S}_{b j}}=-\int_{0}^{T} \int_{\Gamma} E_{b} S_{b u d} \varphi_{j}^{b}(\underline{x}) \frac{\partial \tilde{u}_{x}}{\partial x} \frac{\partial u_{x}}{\partial x} d \Gamma d t-\int_{0}^{T} \int_{\Gamma} \rho_{b} S_{b u d} \underline{u} \underline{\tilde{u}} d \Gamma d t \\
\quad+\epsilon_{1} \alpha_{n 1} \int_{\Gamma}\left[\frac{S_{b}(\underline{x})}{S_{b u d}}-1\right] \varphi_{j}^{b}(\underline{x}) d \Gamma . \\
\frac{\partial J}{\partial \check{E}_{c j}}=-\int_{0}^{T} \int_{\Omega} d \lambda_{D P} \varphi_{j}^{c}(\underline{x}) \operatorname{div}(\underline{u}) \operatorname{div}(\underline{\tilde{u}}) d \Omega d t-\int_{0}^{T} \int_{\Omega} d \mu_{D P} \varphi_{j}^{c}(\underline{x}) \varepsilon(\underline{u}): \mathrm{K}_{c} \varepsilon(\underline{\tilde{u}}) d \Omega d t \\
+\epsilon_{2} \alpha_{n 2} \int_{\Omega}\left[\frac{E_{c}(\underline{x})}{E_{c u d}}-1\right] \varphi_{j}^{c}(\underline{x}) d \Omega .
\end{gathered}
$$

where:

$d \lambda_{D P}=\frac{E_{c u d} v_{c}}{\left(1+v_{c}\right)\left(1-v_{c}\right)}, d \mu_{D P}=\frac{E_{c u d}}{2\left(1+v_{c}\right)}$. 
Once we get the functional gradient, several solves of the direct problem are performed to determine the optimal descent step and we deduce the new set of beam parameters.

\section{Numerical implementation}

The inverse problem is solved in a general way using state of the art finite element softwares. A synthesis of the numerical steps is given in Fig. 2. From a main Python script, we run the finite element software Code_Aster to solve the direct and adjoint problems, which are elastodynamics problems. We specify to Code_Aster Python supervisor to serialize, thanks to Python pickle module, the fields useful for the gradient computation and to export the strain $\left(\varepsilon_{x x}^{s i m}\right)_{i}$ computed at the sensor location $\underline{x}_{i}$. Note that the loading of the adjoint problem corresponds to a 'xx' internal stress $\left(\tilde{\sigma}_{0 x x}\right)_{i}$ in the vicinity of the $\mathrm{i}^{\text {th }}$ sensor location. This loading (see Eq. 5) is given by the data misfit $\delta$ : $\left(\tilde{\sigma}_{0 x x}\right)_{i}(\underline{x}, t)=\left[\left(\varepsilon_{x x}^{\operatorname{sim}}\right)_{i}(t)-\left(\varepsilon_{x x}^{m e s}\right)_{i}(t)\right] \psi_{i}\left(\underline{x}-\underline{x}_{i}\right), i \in\left\{1, \ldots, n_{s}\right\}$.

In practice in Code_Aster, the spatial weight function $\psi_{i}\left(\underline{x}-\underline{x}_{i}\right)$ is constant in the patch of elements associated to the $\mathrm{i}^{\text {th }}$ sensor node. To compute the gradient of the data misfit functional according to Eq. 6 and Eq. 7, for the direct problem, one needs the acceleration $\underline{\ddot{u}}$ in the steel bar and the strain $\varepsilon$ in the steel and the concrete parts. Concerning the adjoint problem, one needs the displacement $\underline{\tilde{u}}$ in the steel bar and the strain $\tilde{\varepsilon}$ in the beam. Using these fields and mesh data, the gradient is computed from a python script. Then, to determine the optimal descent step, which minimizes the data misfit functional, several resolutions of the direct problem are performed using Code_Aster. Once the optimal descent step is obtained, we deduce the new set of beam parameters. The iterative process stops when the data misfit $\delta$ is about the sensor precision.

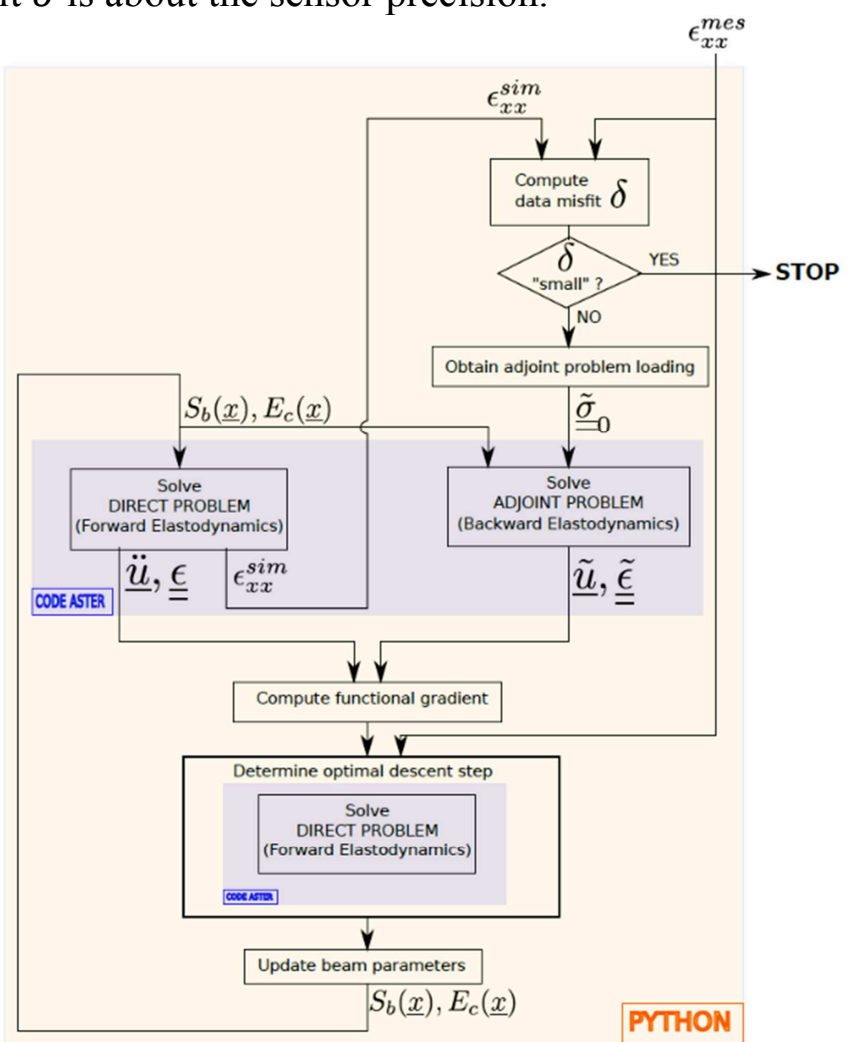

Fig. 2 - Methodology for beam parameter updating

\section{Numerical example}

\section{Definition of the updating problem.}

A 2D concrete beam with a single horizontal steel bar is considered (see Fig. 1 and Fig. 3). Plane strain is assumed. A dynamic load is applied to the top of the concrete beam, it is defined as:

$F_{d}(t)=\left\{\begin{array}{c}-F_{\text {max }} \sin \left(\frac{2 \pi}{T_{c}} t\right) \underline{y}, t \in\left[0, T_{c} / 2\right] . \\ 0, t \in\left[T_{c} / 2, T\right]\end{array}\right.$.

Numerical values: $F_{\max }=10^{4} \mathrm{~N} / \mathrm{m}^{2}, \mathrm{~T}_{\mathrm{c}}=2 \mathrm{~s}, \mathrm{~T}=5 \mathrm{~s}$. 
In this study, the steel bar is decomposed into 5 pieces $\left(n_{b}=5\right.$ in Eq. 1). In each piece $\mathrm{j}$, one has a constant steel bar cross-section $\mathrm{S}_{\mathrm{bj}}$. Concerning the concrete zone, we only consider a loss of Young modulus in the concrete part named $\Omega_{c}$ which is under the steel bar. In the same way, the domain $\Omega_{c}$ is decomposed into 5 subdomains $\left(\mathrm{n}_{\mathrm{c}}=5\right.$ in Eq. 1). In each concrete subdomain $\mathrm{j}$, a constant Young modulus $E_{\mathrm{cj}}$ is taken. We assume that the steel bar cross-section (resp. the concrete Young modulus) is known and undamaged in pieces 1 and 5 (resp. in subdomains 1 and 5): $S_{b 1}=S_{b 5}=S_{b u d}$ (resp. $\left.E_{c 1}=E_{c 5}=E_{c u d}\right)$. As a result, we aim at updating by the inverse modeling technique the normalized steel bar cross-sections and the normalized concrete Young modulus $\check{S}_{b 2}, \check{S}_{b 3}, \check{S}_{b 4}, \breve{E}_{c 2}, \breve{E}_{c 3}, \breve{E}_{c 4}$ defined as:

$S_{b j}=\check{S}_{b j} S_{b u d}$, with $S_{b u d}=0.04 ; E_{c j}=\breve{E}_{c j} E_{c u d}$, with $E_{c u d}=40 G P a$.

To avoid "inverse crime", we take as reference the numerical solution obtained with the finite element code Freefem ++ [7] considering the loading defined in Eq. 10 and the beam parameters:

$\left\{S_{b 1}^{e x}=S_{b 2}^{e x}=S_{b 4}^{e x}=S_{b 5}^{e x}=0.04, S_{b 3}^{e x}=0.03\right.$

$E_{c 1}^{e x}=E_{c 2}^{e x}=E_{c 4}^{e x}=E_{c 5}^{e x}=40 G P a, E_{c 3}^{e x}=30 G P a$

To solve the elastodynamics problem, we take the inconditionally stable Newmark scheme $\left(\gamma=\frac{1}{2}, \beta=1 / 4\right)$. In practice, the data outputs $\varepsilon_{x x}^{m e s}$ are measured. Nevertheless, herein, $\varepsilon_{x x}^{m e s}$ is deduced from the reference numerical solution. We consider 7 strain sensors in the concrete beam, there are located at:

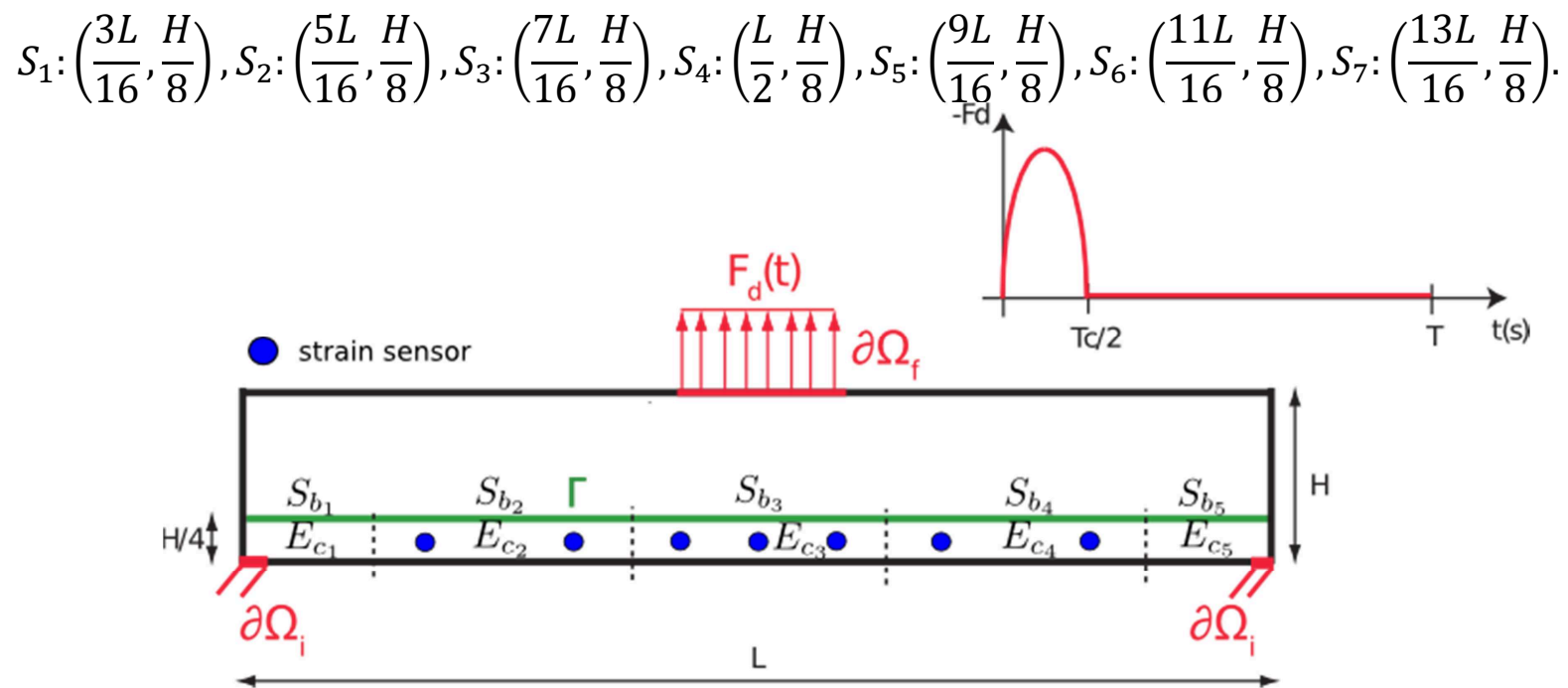

Fig. 3 - Concrete beam with a horizontal steel bar - Steel bar decomposed into 5 pieces. Each piece has a constant cross-section - Lower Concrete part decomposed into 5 subdomains. Each subdomain has a constant Young modulus - Beam instrumented with 7 strains sensors.

Solution of the inverse modeling technique.

The results of the beam identification are given at each iteration of the inverse modeling technique in Table 1. At each iteration of the inverse technique, we evaluate the data misfit $\delta$ and its relative error $\mathrm{E}^{\text {rel }}$ defined by:

$$
\left\{\begin{array}{c}
\delta_{i}=\left(\int_{0}^{T}\left[\left(\varepsilon_{x x}^{\text {sim }}\right)_{i}(t)-\left(\varepsilon_{x x}^{m e s}\right)_{i}(t)\right]^{2} d t\right)^{1 / 2}, i \in\left\{1, \ldots, n_{s}\right\} \\
E_{i}^{r e l}=\frac{\delta_{i}}{\left(\int_{0}^{T}\left[\left(\varepsilon_{x x}^{m e s}\right)_{i}(t)\right]^{2} d t\right)^{1 / 2}}, i \in\left\{1, \ldots, n_{s}\right\}
\end{array}\right.
$$

In Table 1, the value of the maximum data misfit and its associated relative error are given, $\mathrm{k}$ corresponds to the sensor number which has the highest data misfit. 
Table 1 - Steel bar cross-sections and concrete Young modulus at each iteration of the inverse modeling technique

\begin{tabular}{|c|c|c|c|c|c|c|c|c|c|}
\hline & $\mathrm{k}$ & $\delta_{\mathrm{k}}$ & $\mathrm{E}_{\mathrm{k}}{ }^{\text {rel }}[\%]$ & $\mathrm{S}_{\mathrm{b} 2}$ & $\mathrm{~S}_{\mathrm{b} 3}$ & $\mathrm{~S}_{\mathrm{b} 4}$ & $\mathrm{E}_{\mathrm{c} 2}[\mathrm{GPa}]$ & $\mathrm{E}_{\mathrm{c} 3}[\mathrm{GPa}]$ & $\mathrm{E}_{\mathrm{c} 4}[\mathrm{GPa}]$ \\
\hline It 0 & 4 & $2.9 .10^{-7}$ & 10.3 & 0.040 & 0.040 & 0.040 & 40.0 & 40.0 & 40.0 \\
\hline It 1 & 2 & $1.7 .10^{-7}$ & 36.0 & 0.040 & 0.040 & 0.040 & 39.6 & 35.2 & 39.4 \\
\hline It 2 & 2 & $1.6 .10^{-7}$ & 33.7 & 0.040 & 0.039 & 0.040 & 40.0 & 34.1 & 39.4 \\
\hline It 3 & 2 & $1.4 .10^{-7}$ & 28.3 & 0.039 & 0.035 & 0.039 & 40.0 & 31.7 & 40.0 \\
\hline It 4 & 2 & $1.4 .10^{-7}$ & 28.0 & 0.040 & 0.035 & 0.039 & 40.0 & 31.5 & 40.0 \\
\hline
\end{tabular}

We underline the fact that these results are obtained considering "small" regularization parameters $\left(\epsilon_{1}=10^{-6}, \epsilon_{2}=10^{-13}\right)$. After the third iteration, we note that the data misfit reaches a plateau. It corresponds to the error between a numerical solution obtained with FreeFem++ and a numerical solution computed with Code_Aster. As a consequence, the inverse technique should be stopped at iteration 4. At iteration 4 , one has a $28 \%$ error between the ' $x x^{\prime}$ ' strain measured at sensor 2 and the reconstructed strain. This leads to a 5\% error on the identification of the concrete Young modulus in subdomains 3 and to a $15 \%$ error on the identification of the steel bar cross-section in piece 3 . We recall that the reference values of concrete Young modulus and steel bar cross-sections are given in Eq. 12 . We note that the inverse modeling technique enables us to detect the damaged subdomain $\Omega_{\mathrm{c} 3}$ and the damaged interval $\Gamma_{3}$.

\section{Summary}

To identify the beam parameters (steel bar cross-sections and concrete Young modulus), we proposed an inverse modeling technique based on 2D elastodynamics model and dynamic strain responses. On the $2 \mathrm{D}$ concrete beam with a single steel bar, we were able to detect the damaged area. We showed that a $25 \%$ local loss of the steel bar cross-section and a $25 \%$ local decrease of the concrete Young modulus could be detected. It corresponds to a $10 \%$ reduction of the equivalent flexural rigidity. In future works, the inverse modeling technique will be applied to nonlinear dynamics problems.

\section{Acknowledgments}

This research was supported by the region Ile de France. This work was conducted as part of the project "SIPRIS", which involves several partner: Advitam (leader), ASF (Highways of the South of France), CEDRAT, ESIEE Paris, NECS, NEWSTEO, SYROKKO and IFSTTAR.

\section{References}

[1] D. Posenato, P. Kripakaran, D. Inaudi, I. Smith: Methodologies for model-free data interpretation of civil engineering structures, Computers and Structures Vol. 88 (2010), p. 467-482

[2] P. Pankaj, E. Lin: Material modeling in the seismic response analysis for the design of RC framed structures, Engineering Structures Vol. 27 (2005), p. 1014-1023

[3] Eurocode 8: Design of structures for earthquake resistance, Part 2: Bridges, NF EN 1998-2, 2006.

[4] J.L. Lions, Contrôle Optimal des Systèmes Gouvernés par des Equations aux Dérivées Partielles, Dunod, Paris (1961), in french.

[5] Code_Aster, EDF R\&D, Finite element code for structural analysis, GNU general public licence, http://www.code-aster.org .

[6] Salome, CEA/DEN, EDF R\&D, OPEN CASCADE, Open Source Integration Platform for Numerical Simulation, http://www.salome-platform.org .

[7] F. Hecht, O. Pironneau, FreeFEM++, http://www.freefem.org. 